\title{
Controlled Propulsion of Two- Dimensional Microswimmers in a Precessing Magnetic Field
}

\section{Journal Article}

\section{Author(s):}

Tottori, Soichiro; Nelson, Bradley J.

Publication date:

2018-06-14

Permanent link:

https://doi.org/10.3929/ethz-b-000271851

\section{Rights / license:}

Creative Commons Attribution 4.0 International

\section{Originally published in:}

Small 14(24), https://doi.org/10.1002/smll.201800722 


\title{
Controlled Propulsion of Two-Dimensional Microswimmers in a Precessing Magnetic Field
}

\author{
Soichiro Tottori* and Bradley J. Nelson*
}

Magnetically actuated micro-/nanoswimmers can potentially be used in noninvasive biomedical applications, such as targeted drug delivery and micromanipulation. Herein, two-dimensional (2D) rigid ferromagnetic microstructures are shown to be capable of propelling themselves in three dimensions at low Reynolds numbers in a precessing field. Importantly, the above propulsion relies neither on soft structure deformation nor on the geometrical chirality of swimmers, but is rather driven by the dynamic chirality generated by field precession, which allows an almost unconstrained choice of materials and fabrication methods. Therefore, the swimming performance is systematically investigated as a function of precession angle and geometric design. One disadvantage of the described propulsion method is that the fabricated 2D swimmers are achiral, which means that the forward/backward swimming direction cannot be controlled. However, it has been found that asymmetric 2D swimmers always propel themselves toward their longer arm, which implies that dynamic chirality can be constrained to be either right-handed or left-handed by permanent magnetization. Thus, the simplicity of fabrication and possibility of dynamic chirality control make the developed method ideal for applications and fundamental studies that require a large number of swimmers.

connected by DNA, ${ }^{[3]}$ flexible nanowires, ${ }^{[4]}$ or DNA bundles ${ }^{[5]}$ ) and rigid helical structures with magnetic parts/coatings. ${ }^{[6]}$ However, although these swimmers show excellent swimming and control properties, their fabrication processes require highly sophisticated techniques. Another type of magnetic micro- and nanoscopic propulsion is represented by close-tosurface tumbling motion, which results in a net forward movement due to the surface breaking the symmetry of fluid drag around rotating bodies. Tumbling swimmers are simpler to fabricate from the viewpoint of geometry, with notable examples being anisotropic colloids, ${ }^{[7]}$ nanowires, ${ }^{[8]}$ or magnetically assembled particles. ${ }^{[9]}$ However, these swimmers are not capable of swimming away from the surface. Recently, achiral microswimmers comprising three magnetic beads connected by streptavidin-biotin interactions have been reported, ${ }^{[10]}$ being actuated by a rotating magnetic field high enough to induce a wobbling motion for forward pro-

Untethered micro- and nanoswimmers can potentially be used in various biomedical applications, such as drug/cell delivery, remote sensing inside bodies, and cell micromanipulation. ${ }^{[1]}$ At such scales, however, the dominant role of viscosity (as compared to that of inertia) makes swimming more demanding, i.e., the Reynolds number $(R e)$, which reflects the ratio of viscous and inertial forces, falls in the range below unity. ${ }^{[2]}$ Thus, the fluid flow becomes time reversal, and non-reciprocal motion is required to achieve a net forward movement, as typically described by the "scallop theorem."

The non-reciprocal motion of natural swimming microorganisms has inspired the design and fabrication of several types of magnetically actuated artificial micro- and nanoswimmers, including flexible structures (e.g., chains of magnetic colloids

S. Tottori, ${ }^{[+]}$Prof. B. J. Nelson

Institute of Robotics and Intelligent Systems

ETH Zurich

Zurich $\mathrm{CH}-8092$, Switzerland

E-mail: st607@cam.ac.uk; bnelson@ethz.ch

The ORCID identification number(s) for the author(s) of this article can be found under https://doi.org/10.1002/smll.201800722.

${ }^{[+]}$Present address: Cavendish Laboratory, University of Cambridge, CB3 OHE Cambridge, UK

DOI: 10.1002/smll.201800722 pulsion. Similarly, the swimming performance of non-helical randomly shaped structures has also been investigated. ${ }^{[11]}$ However, these achiral swimmers in a rotating field can be either right-handed or left-handed, with the resulting uncontrollability of their swimming direction being a potential problem for a swarm of multiple swimmers.

Herein, we demonstrate the propulsion of two-dimensional (2D) rigid ferromagnetic structures, using a precessing field instead of a rotating field to introduce a non-reciprocal cork-screw motion. Moreover, we correlate swimming velocity with swimmer morphology, i.e., length, angle between arms, number of arms, and field precession angle, also showing that asymmetric 2D structures always swim toward their longer arm due to the hydrodynamic drag-induced stability. The above finding suggests that permanent magnetization of swimmers imparts them with certain magnetotacticity, allowing unidirectional north- or southseeking motion. For practical applications, swimming direction control is important to realize the synchronous motion of multiple swimmers. ${ }^{[12]}$ Due to their simple 2D geometry, the described swimmers can be readily batch-fabricated by conventional lithography from various types of materials that are not easily amenable to $3 \mathrm{D}$ processing. Thus, the $3 \mathrm{D}$ motion of $2 \mathrm{D}$ rigid micro- and nanoswimmers actuated by a precessing field sheds light on the development of magnetic swimming micro/nanorobots without geometrical chirality or undulation-like motion. 
2D swimmers, featuring $\mathrm{Ti} / \mathrm{Ni} / \mathrm{Ti}$ layers with thicknesses of $10 / 180 / 10 \mathrm{~nm}$, respectively, were fabricated using conventional photolithography patterning and electron beam evaporation, with a water-soluble sacrificial layer utilized for release into water. ${ }^{[13]}$ Detailed fabrication and experimental procedures can be found in the Supporting Information. The precessing magnetic field was generated by triaxial orthogonal coil pairs, being defined by field strength $H$, angular velocity $\omega$, and precession angle $\theta$ (Figure 1a). Thus, a precession angle of $90^{\circ}$ corresponded to a normal rotating field, and the precession axis could be changed for steering. Details of the experimental setup are shown in Figure S1 (Supporting Information). The swimmers followed the direction of the magnetic field and propelled themselves along the precession axis, with the symmetry of their shape implying that structures with identical arm lengths could be both right-handed and left-handed (Figure 1a). Time-lapse images of a selected swimmer captured at 1-s intervals shown in Figure 1b (see also Movie S1, Supporting Information) confirmed that the swimming motion was typically diagonal to the direction of the precession axis because of the drift caused by the hydrodynamic wall effect of the bottom surface. ${ }^{[14]}$ When driven vertically, the swimmer moved upward/downward without drift (see Figure S2 and Movie S2, Supporting Information).

We tested various 2D structures having different arm length ratios, angles, and number of arms, with their scanning electron microscopy (SEM) images shown in Figure 1c. The angle $\alpha$ depicted in Figure 1c corresponds to the angle between the easy axis and the bottom arm. The easy axis was estimated by measuring the orientations of the structures in a static magnetic field (5 mT) applied horizontally to the bottom surface, with the results summarized in Table 1 indicating that the above axes roughly coincided with the long axes of the structures. Structure (ii) with a bend angle (angle between two arms) of $60^{\circ}$ had an easy axis of $23^{\circ}$, which was thus pointing between the arms. This finding was ascribed to the switching of the first and second easy axes at low bend angles, in line with the behavior observed for magnetic helices with small and large helix angles. ${ }^{[15]}$ As shown later, this qualitative difference resulted in a completely different swimming behavior, i.e., structure (ii) did not swim stably at all precession angles.

The swimming motion of structure (i) with a bend angle of $90^{\circ}$ (Figure 1c) is characterized in Figure 2. In this case,

a

b

C
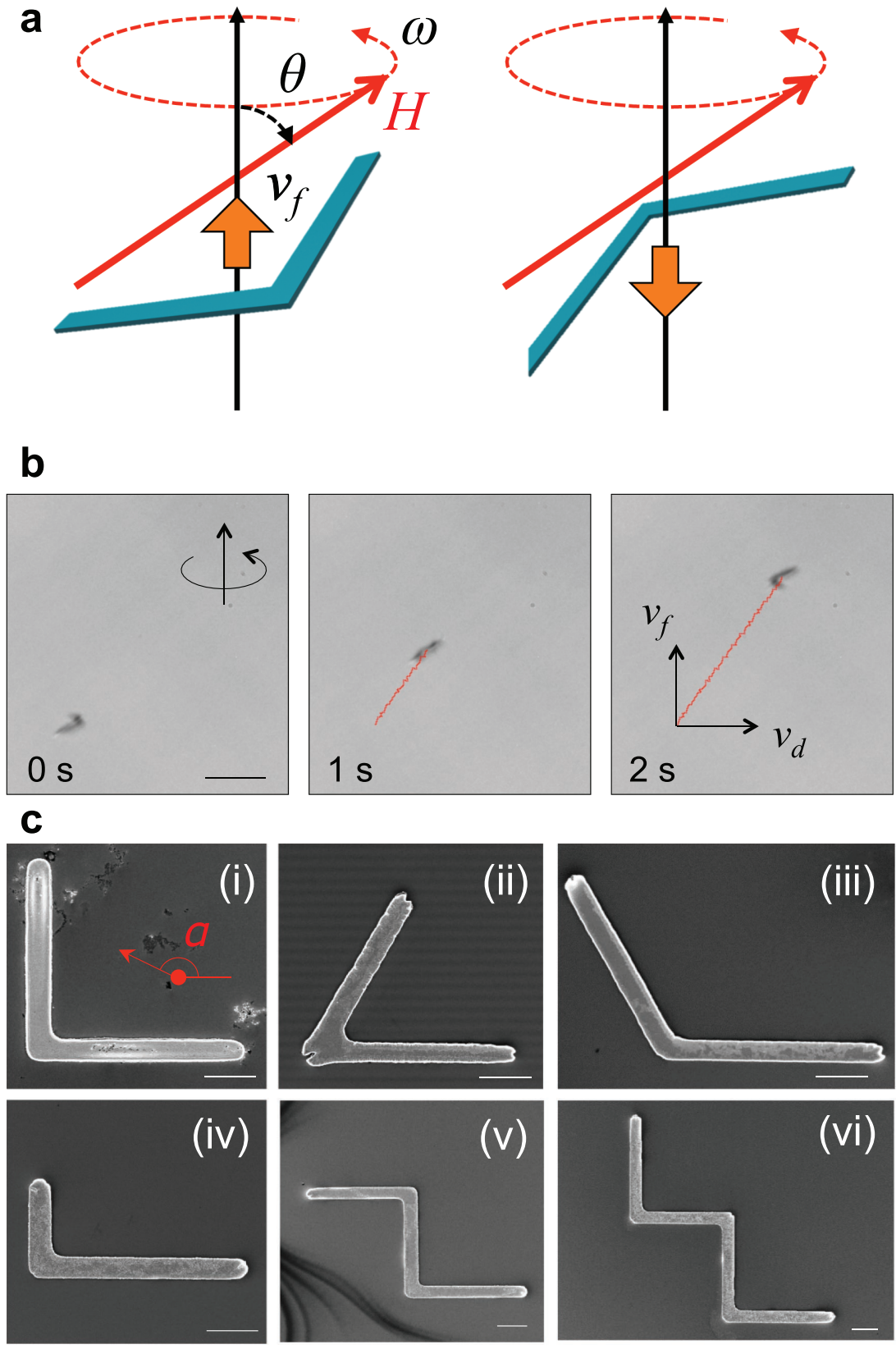

Figure 1. 2D magnetic swimmers. a) Schematic illustrations of 2D swimmers in a precessing field (field strength $H$, angular velocity $\omega$, precession angle $\theta$ ), showing that symmetrical swimmers can be both right-handed and left-handed. Wide orange arrows show swimming directions. b) Time-lapse images of a 2D swimmer in the presence of a precessing field ( $B=5 \mathrm{mT}$, $12 \mathrm{~Hz}$, and $\theta=80^{\circ}$, precession axis indicated by a black arrow), with forward and drift velocities denoted by $v_{\mathrm{f}}$ and $v_{\mathrm{d}}$, respectively. Scale bar equals $50 \mu \mathrm{m}$. c) SEM images of various 2D structures. (i) 2-arm structure $\left(20 \mu \mathrm{m}, 22 \mu \mathrm{m}, 90^{\circ}\right)$, (ii) 2-arm structure $\left(20 \mu \mathrm{m}, 22 \mu \mathrm{m}, 60^{\circ}\right)$, (iii) 2 -arm structure $\left(20 \mu \mathrm{m}, 22 \mu \mathrm{m}, 120^{\circ}\right)$, (iv) 2 -arm structure $\left(10 \mu \mathrm{m}, 22 \mu \mathrm{m}, 90^{\circ}\right)$, (v) 3-arm structure $\left(20 \mu \mathrm{m}, 90^{\circ}\right)$, (vi) 4 -arm structure $\left(20 \mu \mathrm{m}, 90^{\circ}\right)$. The red angle $\alpha$ in (i) indicates the definition of the easy axis of the structure shown in Table 1 . Scale bars equal $5 \mu \mathrm{m}$.

the field strength was fixed at $5 \mathrm{mT}$, and the precession angle and frequency were varied. Both the forward and drift velocities increased linearly with increasing input frequency, indicating that the deviation of swimmer magnetization from the field did not significantly change due to the strong magnetic torque. At precession angles below $65^{\circ}$, the swimmer did not 
Table 1. Direction of the easy axis in Figure 1c, with results given as mean \pm standard deviation.

\begin{tabular}{lcccc}
\hline Shape & (i) $90^{\circ}$ & (ii) $60^{\circ}$ & (iii) $120^{\circ}$ & (iv) 1 to 2 \\
\hline Easy axis angle $\alpha\left[^{\circ}\right]$ & $154.9 \pm 7.1$ & $23.2 \pm 4.3$ & $159.8 \pm 7.4$ & $3.8 \pm 4.8$ \\
\hline
\end{tabular}

synchronize with the field. The forward velocity slope was maximized at a precession angle of $80^{\circ}$, whereas the slope of the drift velocity was maximal at $85^{\circ}$. Previously, the forward propulsion of 2D structures was induced by using the so-called "wobbling regime," in which the precession angle was highly dependent on the field frequency and strength. ${ }^{[10 a]}$ In this case, the wobbling motion was not induced at low frequency, with tumbling motion not resulting in forward propulsion observed instead. However, in a precessing field, the precessing motion of swimmer is introduced not by this wobbling regime, but
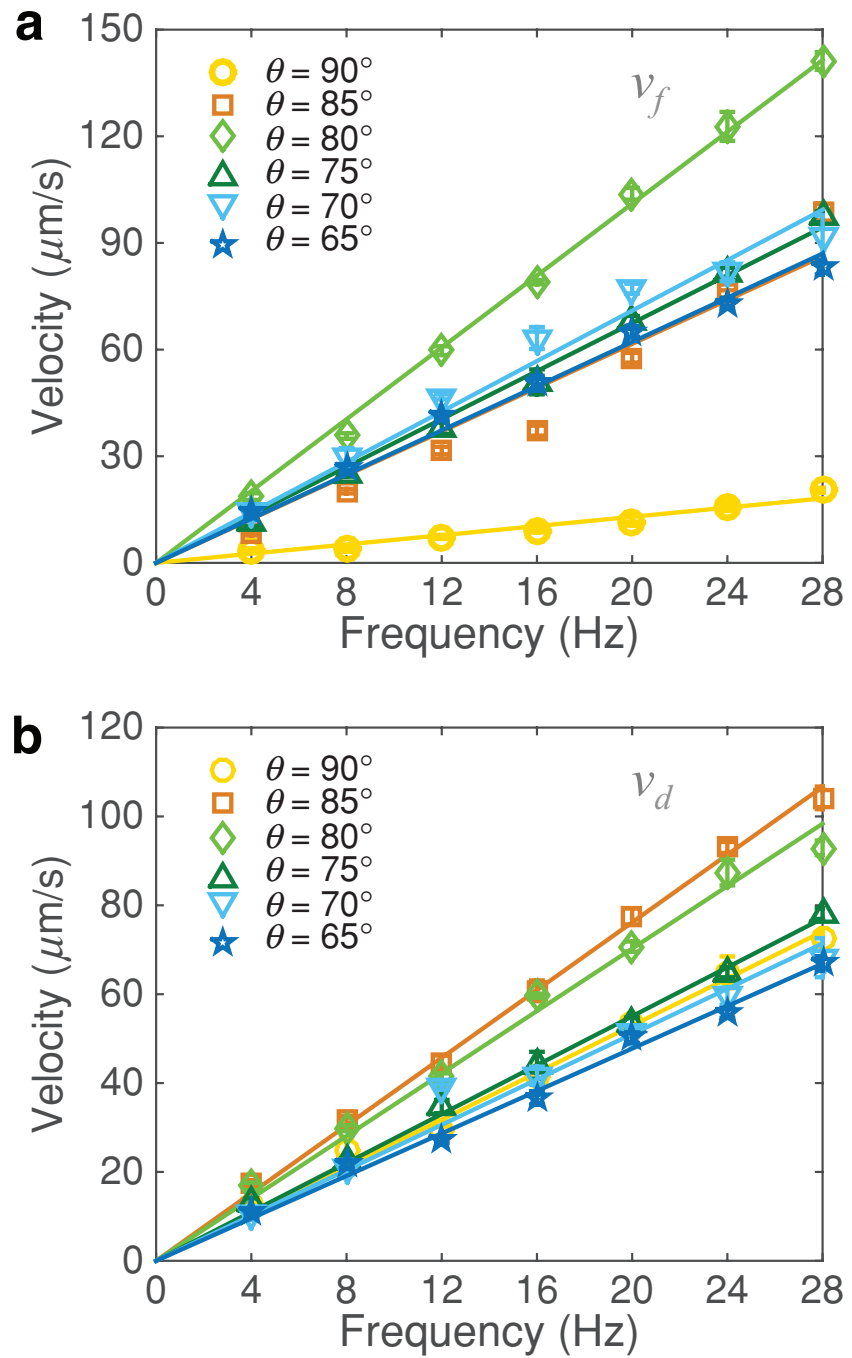

Figure 2. a) Forward and b) drift swimming velocities of swimmer (i) in Figure 1c as functions of input frequency at a fixed field strength $(5 \mathrm{mT})$ and various precession angles $\theta$. For $\theta<65^{\circ}$, the swimmers became unsynchronized with the field. Solid lines represent linear fits to the experimental data, and error bars represent standard deviations from the mean. by the precessing motion of the field itself. Thus, even at low input frequencies, the swimmers can propel themselves in a cork-screw fashion.

To determine the influence of the field precession angle on swimming properties, we examined its effect on fixed-frequency $(20 \mathrm{~Hz}$ ) velocity (Figure 3a,b), revealing an almost complete absence of forward propulsion at $90^{\circ}$ for all geometries, since the induced motion corresponded to simple tumbling. As the precession angle decreased, the forward velocity increased and reached its maximum, with the maximum dimensionless speed $^{[4 \mathrm{~b}]} v_{\mathrm{f}} / L f=0.187\left(v_{\mathrm{f}}=103.8 \mu \mathrm{m} \mathrm{s} \mathrm{s}^{-1}\right.$, body length $L=27.7 \mu \mathrm{m}$, frequency $f=20 \mathrm{~Hz}, \theta=80^{\circ}$ ) realized for swimmer (i) being approximately equal to the highest value reported thus far. ${ }^{[4 b, 11 a]}$ A further decrease of the precession angle reduced the forward swimming velocity and finally resulted in an asynchronous motion, with the transition to the latter mode for swimmers (i), (iii), and (iv) occurring at $65^{\circ}, 50^{\circ}$, and $45^{\circ}$,
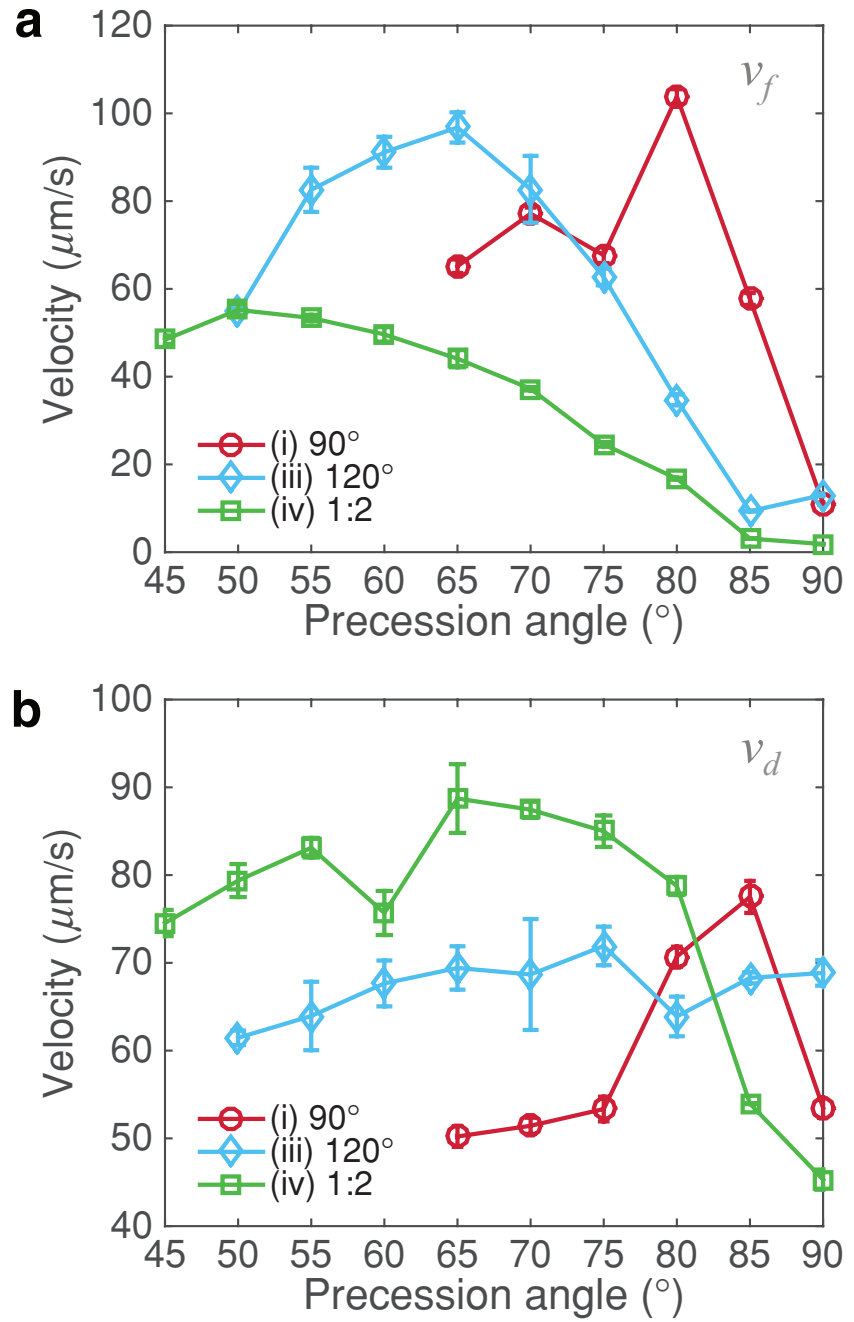

Figure 3. a) Forward and b) drift swimming velocities as functions of the field precession angle obtained at fixed field input frequency $(20 \mathrm{~Hz})$ and strength $(5 \mathrm{mT})$. Swimmers (i), (iii), and (iv) in Figure 1c started to be unsynchronized at precession angles below $65^{\circ}, 50^{\circ}$, and $45^{\circ}$, respectively. Error bars represent standard deviations from the mean. 

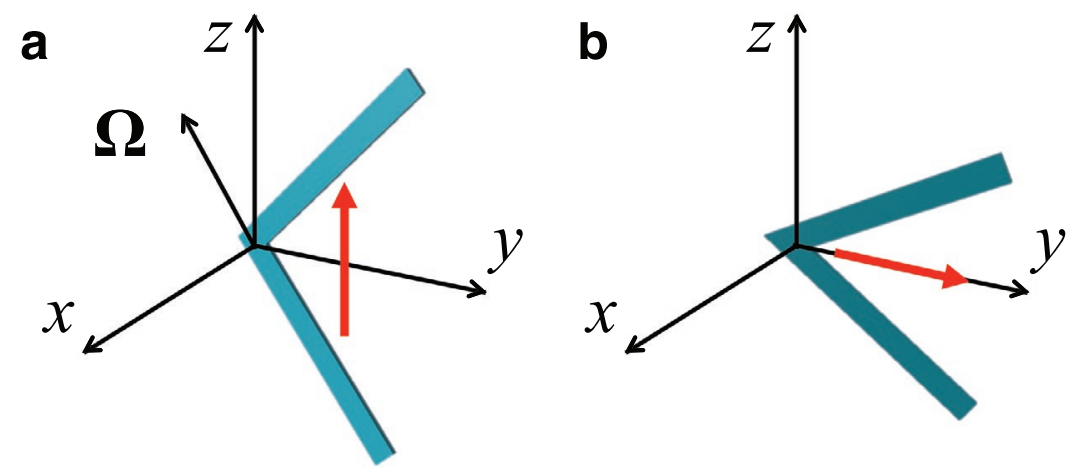

Figure 4. Schematic view of $2 \mathrm{D}$ swimmers with bend angles of a) $90^{\circ}$ and b) $60^{\circ}$ in a Cartesian coordinate system. Red arrows indicate the directions of easy axes.

respectively. The drift velocity also depended on the precession angle (Figure $3 \mathrm{~b}$ ), and the nonmonotonic nature of the obtained curves was ascribed to hydrodynamic interactions with the wall. Structure (ii) with a bend angle $60^{\circ}$ rotated stably only at a precession angle of $90^{\circ}$, undergoing periodic handedness changes and thus not propelling itself forward at other angles, e.g., $85^{\circ}$ (Movie S3, Supporting Information).

In this section, we use a simple analytical approach to show that a precessing field can introduce dynamic chirality into the swimming motion of $2 \mathrm{D}$ structures. In the presence of a magnetic field, a 2D structure exhibits a certain magnetization M. As the field rotates, the magnetic torque is described by $\mathbf{T}=\mu_{0} \mathbf{M} \times \mathbf{H}$, where $\mathbf{H}$ is the external field and $\mu_{0}$ is the permeability of free space. Moreover, the external force $\mathbf{F}$, torque $\mathbf{T}$, translational velocity $\mathrm{V}$, and rotational velocity $\boldsymbol{\Omega}$ are related by ${ }^{[16]}$

$\left[\begin{array}{c}\mathrm{F} \\ \mathrm{T}\end{array}\right]=\left[\begin{array}{cc}\mathrm{A} & \mathbf{B} \\ \mathbf{B}^{\mathrm{T}} & \mathrm{C}\end{array}\right]\left[\begin{array}{l}\mathrm{V} \\ \Omega\end{array}\right]$

The propulsion matrices $\mathbf{A}, \mathbf{B}$, and $\mathbf{C}$ of the $2 \mathrm{D}$ structures in Figure $4 \mathrm{a}, \mathrm{b}$ are given by

$\mathbf{A}=\left[\begin{array}{ccc}A_{1} & 0 & 0 \\ 0 & A_{2} & 0 \\ 0 & 0 & A_{3}\end{array}\right], \mathbf{B}=\left[\begin{array}{ccc}0 & 0 & B_{13} \\ 0 & 0 & 0 \\ B_{31} & 0 & 0\end{array}\right], \mathbf{C}=\left[\begin{array}{ccc}C_{1} & 0 & 0 \\ 0 & C_{2} & 0 \\ 0 & 0 & C_{3}\end{array}\right]$

As there is no external force, $\mathbf{F}=\mathbf{0}$, and the direction of the forward swimming velocity is collinear with the precession axis, allowing the forward swimming speed to be calculated as

$\left|v_{\mathrm{f}}\right|=\frac{|\mathrm{V} \cdot \Omega|}{|\Omega|}=\left|\left(\frac{B_{13}}{A_{1}}+\frac{B_{31}}{A_{3}}\right) \Omega_{1} \Omega_{3}\right|$

which means that the rotational axis must have components in both the $x$ - and $z$-directions, as previously shown by Cheang et al. ${ }^{[10 a]}$ Note that the minimal geometric requirement for the swimmer is actually $\left(B_{13} / A_{1}+B_{31} / A_{3}\right) \neq 0$, not $\mathbf{B} \neq \mathbf{0}$. Herein, we introduced non-zero $\Omega_{1}$ and $\Omega_{3}$ motion by applying a precessing field, which allowed us to tune the wobbling angle independently of the applied rotational frequency. For the symmetrical three-arm structure (v), $\left(B_{13} / A_{1}+B_{31} / A_{3}\right)=0$, whereas the four-arm structure (vi) exhibited non-zero $\left(B_{13} / A_{1}+B_{31} / A_{3}\right)$, similarly to the other two-arm structures. Indeed, the three-arm structure showed nearly zero forward velocity, while the fourarm one exhibited propulsion, as summarized in Table 2. The four-arm structure featured a lower propulsion velocity than the two-arm one, since the inner two arms generated a propulsion force directed oppositely to that generated by the outer two arms. The shift of the velocity peaks in Figure $3 \mathrm{a}$ for the large bend angle can be explained by the fact that the deviation of the precession angle of the swimmer from that of the field decreased with an increasing bend angle. The structure with a narrow bend angle (Figure 4b) has its easy axis on the $y$-axis rather than on the $z$-axis, while the hydrodynamic propulsion matrices (Equation (2)) have the same form. Thus, this qualitative difference can explain the undesired nonpropulsive motion of the structure (ii) in Figure 1c.

Next, we show that the asymmetric swimmer (iv) in Figure 1c always propelled itself toward its longer arm (Figure 5a), since asymmetric 2D structures in rotating/precessing field have a preferential stable direction of rotation. In Figure $5 \mathrm{~b}$, a rotating field $(10 \mathrm{mT}, 2 \mathrm{~Hz})$ was applied with its rotating axis directed to the right side of the figure. The structure was first lifted up by the magnetic torque until $0.15 \mathrm{~s}$, twisting its body and starting to rotate in a long-arm-front direction starting from $0.2 \mathrm{~s}$ (Movie S4, Supporting Information). For symmetric cases (two arms of identical length), no preferential direction exists as long as the magnetization is also symmetric, since both arms generate equal hydrodynamic torques. However, in the case of asymmetric magnetization, the above structures should also exhibit a preferential rotational direction. Thus, if the asymmetric 2D structures are permanently magnetized, as depicted in Figure $5 \mathrm{c}-\mathrm{e}$.g., if the longer arm is magnetized as a north pole-they always rotate in a long-arm-front direction (in this case right-handed) and swim toward the north pole of the precessing field because their orientation and handedness are confined by the magnetic and hydrodynamic torques, respectively. Left-handed rotation (L) is unstable under these conditions and thus flips to righthanded rotation $(\mathrm{R})$, with this pole-seeking behavior mimicking the magnetotaxis of natural north- or south-seeking bacteria. ${ }^{[17]}$ Previously, magnetotactic control of micro- and nanomotors was demonstrated for chemocatalytic and bacterial actuations. ${ }^{[18]}$ However, polar magnetotactic control has not yet been utilized for magnetic actuation to date, since a rotating magnetic field does not have a static pole. The use of a precessing field introduces an extra control parameter for directing magnetic swimmers and can also be used to tune their velocity/frequency ratios by changing the precession angle, potentially increasing the

Table 2. Forward swimming velocities of multiple-arm structures in Figure 1c (i, v, and vi) obtained at a precession angle, input frequency, and field strength of $80^{\circ}, 20 \mathrm{~Hz}$, and $5 \mathrm{mT}$, respectively.

\begin{tabular}{lccc}
\hline Shape & (i) 2-arm & (v) 3-arm & (vi) 4-arm \\
\hline Forward swimming velocity $\nu_{\mathrm{f}}\left[\mu \mathrm{m} \mathrm{s}^{-1}\right]$ & $103.8 \pm 1.7$ & $6.6 \pm 1.0$ & $66.9 \pm 5.5$ \\
\hline
\end{tabular}


a

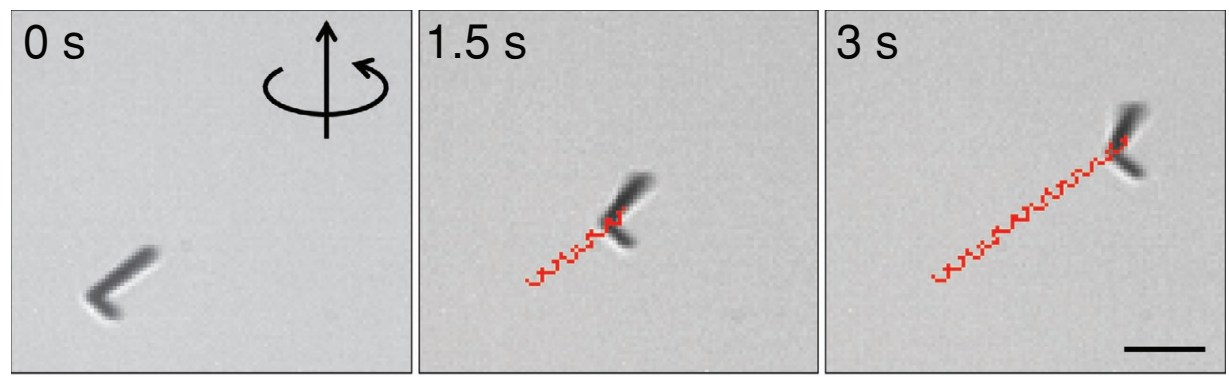

b
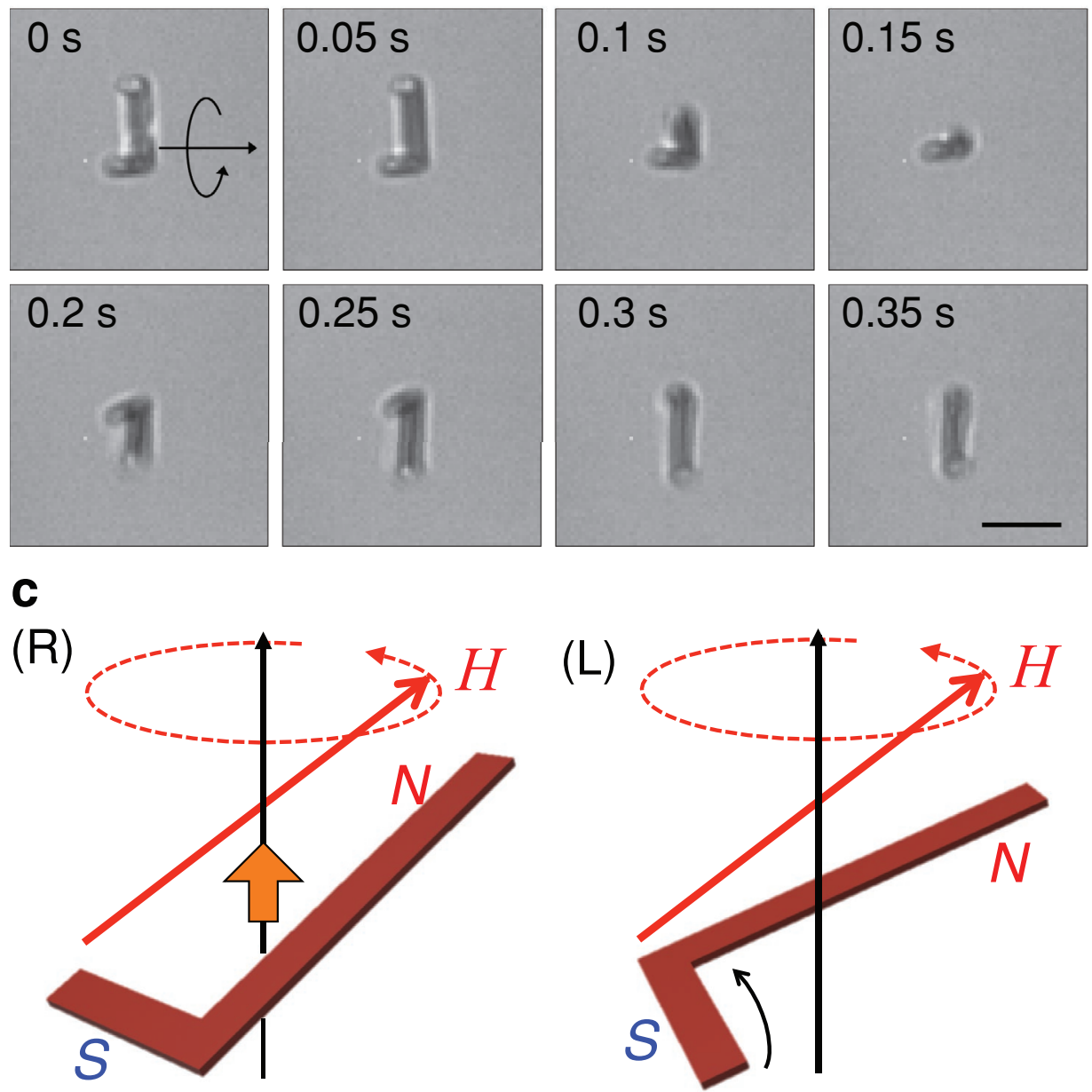

Figure 5. Swimming direction of an asymmetric 2D swimmer. a) An asymmetric structure always swam toward its longer arm (field strength, frequency, and precession angle equaled $5 \mathrm{mT}, 4 \mathrm{~Hz}$, and $55^{\circ}$, respectively). b) Asymmetric 2D structure twisted and aligned to rotate in a long-arm-front direction. A rotating field $\left(10 \mathrm{mT}, 2 \mathrm{~Hz}, \theta=90^{\circ}\right)$ was applied with the rotating axis directed to the right. Scale bars equal $20 \mu \mathrm{m}$. c) Schematic representations of stable and unstable swimming directions of permanently magnetized asymmetric 2D swimmers. Right-handed rotation (R) is stable under these conditions, whereas left-handed rotation $(L)$ is unstable, transforming into right-handed rotation.

variety of control strategies. For example, if a rotating field is applied to a swarm of asymmetric 2D swimmers, one can expect that half of the swimmers will move forward and the rest backward, while switching to a precessing field could result in all swimmers being propelled in the same direction. Thus, further control strategies need to be explored in future studies.
Finally, we discuss the swimming efficiency of the fabricated swimmers. The high dimensionless speed of our 2D swimmer $\left(v_{\mathrm{f}} / L f=0.187\right)$ is largely attributed to the following two factors: 1) The precessing 2D structure moves similarly to a half-helix, with its short body length compared to that of the multihelical structure being advantageous in view of the fact that the 
precession angle is optimized by the field. 2) The cross-section of the filament is perpendicular to the rotation axis, similar to the case of the Archimedes' screw. Indeed, this geometry corresponds to the most efficient cross-section orientation parameter, $\gamma=\pi / 2$, as previously shown for chiral swimmers in a simulation study. ${ }^{[19]}$ Another interesting aspect of a precessing field is that it can tune the rotation-translation coupling rate. In a Newtonian fluid, the dynamics of the above magnetic swimmers can be optimized at certain precession angles. However, in the cases of biological or biomedical applications, the surrounding media contain tissue or a heterogeneous and viscoelastic polymer mesh, ${ }^{[20]}$ making the possibility of on-demand precession angle adjustment useful for adapting and optimizing swimming behavior in these situations.

In summary, we have described the fabrication of simple 2D swimmers and assessed their swimming mechanism in a precessing field, showing that the resulting precessing motion allows these swimmers to gain dynamic chirality along the precession axis and swim forward in a cork-screw fashion. Simple analysis suggested that these 2D swimmers can indeed convert rotational motion into translational motion, additionally revealing that their swimming efficiency can be tuned by adjusting the precession angle. Importantly, asymmetric 2D swimmers were found to always swim toward their longer arms, with permanent magnetization allowing one to make all swimmers either north- or south-seeking. Thus, the proposed method broadens the material and design scope of microscopic swimmers while allowing unidirectional motion control that is essential for swarm steering. These features allow the 2D swimmers to be used as simple alternatives to helical/flexible swimmers for in vivo targeted drug delivery or micromanipulation.

\section{Supporting Information}

Supporting Information is available from the Wiley Online Library or from the author.

\section{Acknowledgements}

The authors thank L. Zhang for discussions and the FIRST lab at ETH Zurich for technical support.

\section{Conflict of Interest}

The authors declare no conflict of interest.

\section{Keywords}

chirality, low Reynolds number, microswimmers, nanomotors, precessing magnetic fields

Received: February 21, 2018

Revised: March 20, 2018

Published online: May 10, 2018
[1] a) B. J. Nelson, I. K. Kaliakatsos, J. J. Abbott, Annu. Rev. Biomed. Eng. 2010, 12, 55; b) S. Tottori, L. Zhang, B. J. Nelson, in Nanomedicine, Springer, New York, NY 2014, p. 171; c) J. Li, B. Esteban-Fernández de Ávila, W. Gao, L. Zhang, J. Wang, Sci. Rob. 2017, 2, eaam6431; d) H. Wang, M. Pumera, Chem. Rev. 2015, 115, 8704; e) W. T. Duan, W. Wang, S. Das, V. Yadav, T. E. Mallouk, A. Sen, Annu. Rev. Anal. Chem. 2015, 8, 311; f) H. Ceylan, J. Giltinan, K. Kozielski, M. Sitti, Lab Chip 2017, 17, 1705.

[2] a) E. M. Purcell, Am. J. Phys. 1977, 45, 3; b) C. Bechinger, R. Di Leonardo, H. Lowen, C. Reichhardt, G. Volpe, G. Volpe, Rev. Mod. Phys. 2016, 88, 045006.

[3] R. Dreyfus, J. Baudry, M. L. Roper, M. Fermigier, H. A. Stone, J. Bibette, Nature 2005, 437, 862.

[4] a) W. Gao, S. Sattayasamitsathit, K. M. Manesh, D. Weihs, J. Wang, J. Am. Chem. Soc. 2010, 132, 14403; b) O. S. Pak, W. Gao, J. Wang, E. Lauga, Soft Matter 2011, 7, 8169; c) B. Jang, E. Gutman, N. Stucki, B. F. Seitz, P. D. Wendel-Garcia, T. Newton, J. Pokki, O. Ergeneman, S. Pane, Y. Or, B. J. Nelson, Nano Lett. 2015, 15, 4829.

[5] A. M. Maier, C. Weig, P. Oswald, E. Frey, P. Fischer, T. Liedl, Nano Lett. 2016, 16, 906.

[6] a) L. Zhang, J. J. Abbott, L. X. Dong, B. E. Kratochvil, D. Bell, B. J. Nelson, Appl. Phys. Lett. 2009, 94, 064107; b) A. Ghosh, P. Fischer, Nano Lett. 2009, 9, 2243; c) S. Tottori, L. Zhang, F. Qiu, K. K. Krawczyk, A. Franco-Obregón, B. J. Nelson, Adv. Mater. 2012, 24, 811; d) M. Medina-Sanchez, L. Schwarz, A. K. Meyer, F. Hebenstreit, O. G. Schmidt, Nano Lett. 2016, 16, 555; e) K. E. Peyer, S. Tottori, F. M. Qiu, L. Zhang, B. J. Nelson, Chem. Eur. J. 2013, 19, 28.

[7] a) P. Tierno, R. Golestanian, I. Pagonabarraga, F. Sagues, Phys. Rev. Lett. 2008, 101, 218304; b) M. Driscoll, B. Delmotte, M. Youssef, S. Sacanna, A. Donev, P. Chaikin, Nat. Phys. 2017, 13, 375.

[8] L. Zhang, T. Petit, Y. Lu, B. E. Kratochvil, K. E. Peyer, R. Pei, J. Lou, B. J. Nelson, ACS Nano 2010, 4, 6228.

[9] C. E. Sing, L. Schmid, M. F. Schneider, T. Franke, A. Alexander-Katz, Proc. Natl. Acad. Sci. USA 2010, 107, 535.

[10] a) U. K. Cheang, F. Meshkati, D. Kim, M. J. Kim, H. C. Fu, Phys. Rev. E 2014, 90, 033007; b) F. Meshkati, H. C. Fu, Phys. Rev. E 2014, 90, 063006.

[11] a) P. J. Vach, P. Fratzl, S. Klumpp, D. Faivre, Nano Lett.. 2015, 15, 7064; b) K. I. Morozov, Y. Mirzae, O. Kenneth, A. M. Leshansky, Phys. Rev. Fluids 2017, 2, 044202.

[12] S. Tottori, L. Zhang, K. E. Peyer, B. J. Nelson, Nano Lett. 2013, 13, 4263.

[13] V. Linder, B. D. Gates, D. Ryan, B. A. Parviz, G. M. Whitesides, Small 2005, 1, 730.

[14] W. R. DiLuzio, L. Turner, M. Mayer, P. Garstecki, D. B. Weibel, H. C. Berg, G. M. Whitesides, Nature 2005, 435, 1271.

[15] K. I. Morozov, A. M. Leshansky, Nanoscale 2014, 6, 12142.

[16] E. Lauga, T. R. Powers, Rep. Prog. Phys. 2009, 72, 096601.

[17] a) R. Blakemore, Science 1975, 190, 377; b) D. Faivre, D. Schuler, Chem. Rev. 2008, 108, 4875

[18] a) G. J. Zhao, S. Sanchez, O. G. Schmidt, M. Pumera, Chem. Commun. 2012, 48, 10090; b) O. Felfoul, M. Mohammadi, S. Taherkhani, D. de Lanauze, Y. Z. Xu, D. Loghin, S. Essa, S. Jancik, D. Houle, M. Lafleur, L. Gaboury, M. Tabrizian, N. Kaou, M. Atkin, T. Vuong, G. Batist, N. Beauchemin, D. Radzioch, S. Martel, Nat. Nanotechnol. 2016, 11, 941.

[19] E. E. Keaveny, S. W. Walker, M. J. Shelley, Nano Lett. 2013, 13, 531.

[20] a) B. J. Nelson, K. E. Peyer, ACS Nano 2014, 8, 8718; b) D. Walker, B. T. Kasdorf, H. H. Jeong, O. Lieleg, P. Fischer, Sci. Adv. 2015, 1, e1500501. 